\title{
Septal implantation of the Micra transcatheter pacing system guided by intraprocedural transesophageal echocardiography
}

\author{
Krzysztof Kaczmarek¹, Iwona Cygankiewicz¹, Bartosz Czarniak², Piotr Jakubowski', \\ Aleksander Strzelecki', Jerzy K. Wranicz', Jarosław Drożdż², Paweł Ptaszyński² \\ 1 Department of Electrocardiology, Medical University of Lodz, Łódź, Poland \\ 2 Department of Cardiology, Medical University of Lodz, Łódź, Poland
}

Correspondence to: Krzysztof Kaczmarek, MD, PhD, Department of Electrocardiology, Medical University of Lodz, ul. Pomorska 251 92-213 Łódź, Poland phone: +48422014360, email: krzysztof.kaczmarek@umed.lodz.pl Received: August 2, 2019. Revision accepted: October 30, 2019. Published online: October 30, 2019. Kardiol Pol. 2019; 77 (12): 1190-1192 doi:10.33963/KP.15043

Copyright by the Author(s), 2019
Introduction Clinical experience with leadless pacemakers is still limited, and safety issues remain an important concern for clinicians. Septal positioning of a leadless pacemaker might be safer in terms of reducing the risk of heart wall perforation. ${ }^{1-3}$

Fluoroscopic guidance is routinely used for implantation of conventional pacemakers. It is also used for leadless pacing systems; however, its inadequacy in the precise positioning of leads has been reported. ${ }^{4}$ Transesophageal echocardiography (TEE) has an established value in guiding several procedures in invasive cardiology. ${ }^{5}$ Therefore, we aimed to determine the usefulness of this technique in guiding septal implantation of the Micra transcatheter pacing system (TPS) (Medtronic, Minneapolis, United States).

Methods This single-center prospective observational study included the first 24 consecutive patients who underwent an implantation of the Micra TPS. The septal position of the pacemaker was considered optimal to reduce the risk of heart perforation. Fluoroscopic guidance with contrast media injection was used according to the standard of care in our center, including single-plane fluoroscopy in at least 3 complementary projections. Subsequently, in all patients, the septal positioning of the Micra device achieved with fluoroscopy was confirmed with intraprocedural TEE (Vivid 9, GE Healthcare, Salem, Connecticut, United States). Local anesthesia together with intravenous conscious sedation (midazolam and fentanyl) was used in 23 patients. General anesthesia was applied in 1 patient due to predicted long duration of the procedure. Three TEE views: $0^{\circ}$ lower-mid-esophageal, $120^{\circ}$ upper-mid-esophageal, and transgastric were selected for visualization of the right ventricle (RV) and were further investigated. Similar to other studies, ${ }^{6}$ the usefulness of particular TEE views was evaluated with a self-developed 3-grade score (TABLE 1). Additionally, the angle between the long axis of the Micra device and the long axis of the interventricular septum, herein termed "deployment angle," was evaluated on TEE. All patients underwent transthoracic echocardiography, chest radiography, and device interrogation on the postprocedural day. The clinical course as well as the electrical parameters of the Micra TPS were evaluated during follow-up visits.

The study was conducted according to the Declaration of Helsinki and was approved by the Bioethical Committee of the Medical University of Lodz (Łódź, Poland). Each patient gave written informed consent before undergoing the implantation procedure.

Statistical analysis Statistical analysis was performed using the Statistica software (StatSoft Inc., Tulsa, Oklahoma, United States). The $t$ test or Mann-Whitney test was used for between-group comparisons of continuous variables according to data distribution. The $\mathrm{X}^{2}$ test and its modification were used to compare categorical data. A $P$ value of less than 0.05 was considered significant.

Results and discussion The study group included 15 men (63\%) and 9 women (mean [SD] 
TABLE 1 Transesophageal echocardiography scoring

\begin{tabular}{lllll} 
TEE projection & Score 0 & Score 1 & Score 2 & P value \\
\cline { 1 - 4 } TEE-LME- $00^{\circ}$ & 2 & 11 & 11 & $<0.01^{\mathrm{a}}$ \\
\cline { 1 - 4 } TEE-UME-120 & 0 & 5 & 19 & $<0.01^{\mathrm{b}}$ \\
\cline { 1 - 4 } TEE-TG & 10 & 10 & 4 & \\
\hline
\end{tabular}

a For the $\chi^{2}$ for the $3 \times 3$ table

b For the $\chi^{2}$ for the $3 \times 2$ table (score 2 vs $<2$ )

Scoring system: 0 , images that did not visualize the Micra device and therefore could not be used to support the procedure; 1 , images of moderate quality that visualized the device but not precisely enough to guide implantation; 2 , the Micra device visualized in detail in relation to the free wall of the right ventricle and interventricular septum

Abbreviations: LME, lower-mid-esophageal; TEE, transesophageal echocardiography; TG transgastric; UME, upper-mid-esophageal

age, 69.7 [18] years; range, 29-95 years). Stable coronary artery disease was reported in $29 \%$ of patients. Two patients had reduced left ventricular ejection fraction and were diagnosed with congestive heart failure. Approximately half of the study group (54.2\%) presented with chronic renal failure. Atrioventricular blocks with sinus rhythm were found to be the most common indication for permanent pacing (58.3\%), followed by bradycardia associated with chronic atrial tachyarrhythmia (29.2\%) and by sinus node dysfunction (12.5\%). None of the patients in sinus rhythm developed pacemaker syndrome after implantation. The main reason for the selection of a leadless system, reported in 22 individuals (91.8\%), was an increased risk of infection related to conventional pacemakers, which resulted from chronic hemodialysis (41.6\%), history of previous device-related infection (33.3\%), chronic infections (20.8\%), or long-term high-dose steroid therapy (8.3\%).

Transesophageal echocardiography images appropriate for guidance of the device positioning were obtained in all patients. Mid-esophageal probe positions, especially upper-mid-esophageal, were more useful than the transgastric one, and a 3-dimensional (3D) imaging facility did not provide any additional benefit. For the lower-mid-esophageal projection, the mean (SD) score was 1.4 (0.7) for 2-dimensional (2D) imaging, as compared with 0.8 (0.5) for 3D imaging $(P<0.01)$. For the upper-mid-esophageal projection, the mean (SD) score for 2D and 3D imaging was $1.8(0.4)$ and $0.9(0.5)$, respectively $(P<0.01)$. Finally, for the transgastric projection, the mean (SD) score for $2 \mathrm{D}$ and 3D imaging was $0.8(0.7)$ and $0.2(0.4)$, respectively $(P<0.01)$.

The septal position (50\% mid and 50\% low septum) of the device was achieved in all $24 \mathrm{pa}-$ tients. Transesophageal echocardiography allowed a verification of the device positioning in 10 cases (41.6\%), in which the initial septal position selected based on fluoroscopy actually appeared to be on the free wall of the RV. Subsequently, the device was relocated to the interventricular septum. In these cases, nonseptal position of the device was characterized by a more acute deployment angle (mean [SD], 18.8 ${ }^{\circ}$ [6.4 $\left.{ }^{\circ}\right]$ vs $\left.27.0^{\circ}\left[8.4^{\circ}\right] ; P<0.01\right)$.

The mean (SD) duration of implantations was 74.6 (34.9) minutes, with a mean (SD) required duration of fluoroscopy of 7.9 (5.2) minutes. The procedures resulted in optimal electrical parameters in all cases (mean [SD] capture threshold, 0.51 [0.23] V; sensing, 11.5 [4.8] mV; and impedance, 703 [140] ohm). Although we did not observe any anatomical signs of nonoptimal TEE-guided septal TPS deployment, in 9 patients (37.2\%), the device had to be repositioned at least once to achieve optimal electrical parameters. The rate of repositions was similar to that reported by other authors. ${ }^{7,8}$

Periprocedural complications were not observed. In all patients, intraprocedural and in-hospital electrical parameters of the pacing system were within the normal range. In-hospital observation was uneventful in all patients. During the mean (SD) follow-up of 418 (224) days, 1 patient died of noncardiovascular cause and another one developed gradual elevation of pacing threshold that did not require an intervention.

The first-in-human experience with leadless pacemakers was reported in 2014, summarizing implantations of these devices in 33 patients. ${ }^{9}$ In that study, 1 patient had cardiac tamponade that resulted in death despite a successful chest surgery. Subsequent studies described low rates of pericardial effusion or tamponade (observed in $1 \%$ to $2 \%$ of cases). ${ }^{7,10}$ Nevertheless, some authors reported a higher prevalence of this complication. ${ }^{2,3,11}$ The septal positioning of the Micra device was suggested to have a favorable effect on the risk of perforation. ${ }^{7,8}$ However, in all relevant studies, devices were implanted mainly into the RV apex. , $7,10,12$ Suprisingly, in 4 of the 5 patients with Micra-related pericardial effusion or tamponade, the devices were implanted in the septal position. ${ }^{12}$ In a study by Kaczmarek et al, ${ }^{3}$ who investigated septal implantations of Micra devices, a single heart perforation occurred when a delivery sheath was directed to the free wall of the RV. ${ }^{3}$

The above examples as well as the experience with conventional pacemakers suggest that fluoroscopy might be insufficient for guiding a precise lead or device implantation in the interventricular septum, at least in some cases. Therefore, in these cases, an additional use of echocardiography seems to be advisable. ${ }^{4,13}$ In our experience, the upper-mid-esophageal position of the TEE probe appeared to be the best option for most patients. Similar views are used for other cardiovascular interventions within the interventricular septum. ${ }^{14}$ According to our results, 3-dimensional imaging facility offers no advantage 
over standard 2-dimentional imaging. This is in line with a report by Faletra et $\mathrm{al},{ }^{14}$ and it probably results from a higher number of artifacts in the case of the 3D technique.

Our main finding is that the septal positioning of the Micra device can be misdiagnosed in $40 \%$ of patients. Therefore, we recommend using TEE on top of fluoroscopy when planning septal implantation of the device. The main limitation of our study is the lack of randomization and a relatively small number of patients. Therefore, our findings should be confirmed in larger studies.

\section{ARTICLE INFORMATION}

CONFLICT OF INTEREST KK and PP received lecturer, educational, and travel grants from Medtronic. Other authors declare no conflict of interest.

OPEN ACCESS This is an Open Access article distributed under the terms of the Creative Commons Attribution-NonCommercial-NoDerivatives $4.0 \mathrm{In}$ ternational License (CC BY-NC-ND 4.0), allowing third parties to download articles and share them with others, provided the original work is properly cited, not changed in any way, distributed under the same license, and used for noncommercial purposes only. For commercial use, please contact the journal office at kardiologiapolska@ptkardio.pl.

HOW TO CITE Kaczmarek K, Cygankiewicz I, Czarniak B, et al. Septal implantation of the Micra transcatheter pacing system quided by intraprocedural transesophageal echocardiography. Kardiol Pol. 2019; 77: 1190-1192. doi:10.33963/ KP.15043

\section{REFERENCES}

1 Martínez-Sande JL, García-Seara J, Rodríguez-Mañero M, et al. The Micra Leadless Transcatheter Pacemaker. Implantation and mid-term follow-up results in a single center. Rev Española Cardiol. 2017; 70: 275-281.

2 Hai JJ, Fang J, Tam CC, et al. Safety and feasibility of a mid-septal implantation technique of a leadless pacemaker. Heart Rhythm. 2019; 16: 896-902.

3 Kaczmarek K, Czarniak B, Jakubowski $P$, et al. Leadless pacemaker and subcutaneous implantable cardioverter-defibrillator therapy: the first use of a novel treatment option in Poland. Kardiol Pol. 2018; 76: 1026.

4 Sharma G, Salahuddin S, Sanders P, et al. Inadequacy of fluoroscopy and electrocardiogram in predicting septal position in RVOT pacing - validation with cardiac computed tomography. Indian Heart J. 2016; 68: 174-180.

5 Hahn RT, Abraham T, Adams MS, et al. Guidelines for performing a comprehensive transesophageal echocardiographic examination: recommendations from the American Society of Echocardiography and the Society of Cardiovascular Anesthesiologists. Anesth Analg. 2014; 118: 21-68.

6 Baran J, Stec S, Pilichowska-Paszkiet E, et al. Intracardiac echocardiography for detection of thrombus in the left atrial appendage comparison with transesophageal echocardiography in patients undergoing ablation for atrial fibrillation: the Action-ICE I study. Circ Arrhythmia Electrophysiol. 2013; 6: 1074-1081.

7 Reddy VY, Exner DV, Cantillon DJ, et al. Percutaneous implantation of an entirely intracardiac leadless pacemaker. N Engl J Med. 2015; 373: 1125-1135.

8 Bonner M, Eggen M, Haddad T, et al. Early performance and safety of the Micra Transcatheter Pacemaker in pigs. Pacing Clin Electrophysiol. 2015; 38: 1248-1259.

9 Reddy VY, Knops RE, Sperzel J, et al. Permanent leadless cardiac pacing: Results of the LEADLESS trial. Circulation. 2014; 129: 1466-1471.

10 Duray GZ, Ritter P, El-Chami M, et al. Long-term performance of a transcatheter pacing system: 12-month results from the Micra Transcatheter Pacing Study. Heart Rhythm. 2017; 14: 702-709.

11 Vamos M, Erath JW, Benz AP, et al. Incidence of cardiac perforation with conventional and with leadless pacemaker systems: a systematic review and meta-analysis. J Cardiovasc Electrophysiol. 2016; 28: 336-346.

12 Roberts PR, Clementy N, Al Samadi F, et al. A leadless pacemaker in the real-world setting: the Micra Transcatheter Pacing System Post-Approval Registry. Heart Rhythm. 2017; 14: 1375-1379.

13 Squara F, Scarlatti D, Riccini P, et al. Individualized left anterior oblique projection. A highly reliable patient-tailored fluoroscopy criterion for right ventricular lead positioning. Circ Arrhythm Electrophysiol. 2018; 11: e006107.

14 Faletra FF, Ramamurthi A, Dequarti MC, et al. Artifacts in three-dimensional transesophageal echocardiography. J Am Soc Echocardiogr. 2014; 27: 453-462. 